\title{
Combinatorial Design of the MAUT and PAMSSEM II Methods for Multiple Attributes Group Decision Making with Probabilistic Linguistic Information
}

\section{Qiaoyu Kong}

Business School, Sichuan Normal University, Chendu, China

Liangping Wu ( $\nabla$ wuliangping6@sina.com )

Business School, Sichuan Normal University, Chengdu, China

\section{Research Article}

Keywords: Probabilistic linguistic term set, Hesitant probabilistic fuzzy set, PL-MAUT-PAMSSEM II method, Multiple attributes group decision making

Posted Date: January 3rd, 2022

DOI: https://doi.org/10.21203/rs.3.rs-1214824/v1

License: (c) (i) This work is licensed under a Creative Commons Attribution 4.0 International License.

Read Full License 


\section{Combinatorial design of the MAUT and PAMSSEM II methods for multiple attributes group decision making with probabilistic linguistic information}

1. Business School, Sichuan Normal University, Chengdu, China.

2. V.C. \& V.R. Key Lab, Sichuan Normal University, Chengdu, China.

Qiaoyu Kong ${ }^{1,2}$ is currently pursuing the master's degree with the Business School, Sichuan Normal University, Chengdu, China. Her research research interests include decision theory and method, statistical analysis, and management science.

Email:1938640307@qq.com, Tel: +17784287513.

Address: No.1819, 2rd section of Chenglong Avenue, Longquanyi District, Chengdu City, China

Liangping $\mathbf{W} \mathbf{u}^{1,2}$ received the M.Sc. and Ph.D. degrees in applied mathematics from the College of Mathematics and Software Science, Sichuan Normal University, Chengdu, China. From 2017 to 2019, he was a Post-Doctoral Researcher in management science and engineering with the School of Economics and Management, Southeast University, Nanjing, China. He is currently an Associate Professor with the Business School, Sichuan Normal University. His research interests include decision theory and method, statistical analysis, and management science.

Email: wuliangping6@sina.com, Tel: +13438137368.

Address: No.1819, 2rd section of Chenglong Avenue, Longquanyi District, Chengdu City, China

Corresponding author: Liangping Wu (wuliangping6@sina.com) 


\title{
Combinatorial design of the MAUT and PAMSSEM II methods for multiple attributes group decision making with probabilistic linguistic information
}

\begin{abstract}
This paper considers the application of probabilistic linguistic term sets (PLTS) in multiple-attribute group decision-making (MAGDM) when the weights can't be determined. First, as an improvement of the PROMETHEE method, the PAMSSEM method can not only handle missing evaluations, but also proposes a rejection threshold to calculate the overall consistency of the plan, so as to rank the plan more reasonably. At the same time, the MAUT uses the marginal utility function to reallocate the attribute values of the alternatives in the interval $[0,1]$, and then calculate the total utility to sort them. Because the utility function is beneficial in expressing consumer satisfaction, we combine the MAUT method and PAMSSEM II method and apply it to solve decision-making problems under probabilistic linguistic environment. Secondly the coefficient of variation method, entropy method and analytic hierarchy process are used to calculate the weights in a combination. In the process of data processing, we use the transfer function to convert the PLTS into the hesitant probabilistic fuzzy set (HPFS) for calculation. Finally, the PL-MAUT-PAMSSEM II method, PROMRTHE method, TOPSIS method and ARAS method are compared with each other.
\end{abstract}

Keywords Probabilistic linguistic term set · Hesitant probabilistic fuzzy set · PL-MAUT-PAMSSEM II method • Multiple attributes group decision making

\section{Introduction}

Decision-making activities are pervasive in all aspects of our lives. The decision-making process includes asking questions, determining alternatives, choosing alternatives, and making decisions (Xu and Zhang 2021). Due to the diversity of decision-making problems and the ambiguity of human thinking, it is not easy for us to use specific numbers to accurately describe the value of each alternative under different standards when making assessments 
(Saleem and NoorUl 2014). Therefore, obtaining accurate assessments requires high costs. In the MAGDM problem, the decision makers (DMs) are required to provide the evaluation value of different alternatives under multiple attributes. Because it is difficult to provide accurate numbers to express preferences, linguistic terms such as "medium" or "good" are used as evaluation information (He et al. 2016; Ji and Yu 2017; Lin et al. 2019; Qu et al. 2018; Sun et al. 2019; Xi et al. 2019; Xi et al. 2018). Therefore, more and more scholars are aware of the importance of probability information in decision-making compared with digital information and devote themselves to related research (Wang et al. 2018b).

Based on the above situation, Zadeh (1975) introduced the Linguistic Term Set (LTS), which expresses DM's preferences through one or more linguistic information, and uses fuzzy linguistic to model qualitative evaluation information. Since then, scholars have carried out further research based on LTS, including linguistic cloud information (Herrera et al. 2000), two-tuple linguistic model (Xu 2004), uncertain linguistic structure (Herrera et al. 2008), unbalanced linguistic information (Xu 2008), and unbalanced uncertain linguistic information (Wang et al. 2014).

With the enrichment of human practical activities, the difficulty of decision-making is getting bigger, and some models that have been proposed cannot completely and accurately express the preferences of DMs in the actual decision-making problem (Wang et al. 2018b). For example, when a DM evaluates alternative plans for inbound tourist attractions, he cannot be $100 \%$ sure that the plan is "good", or he may think it is "medium", "good" or "very good". To help DMs make more reasonable decisions, Rodriguez et al. (2012) proposed the concept of hesitating fuzzy linguistic term set (HFLTS), and Liao et al. (2015) introduced the mathematical form of HFLTS, which allows the DMs to hesitate between several linguistic terms.

However, HFLTS believes that different linguistic terms have the same importance or weight, which is likely to be inconsistent with the true wishes of the DMs. For example, suppose there are 10 DMs evaluating alternatives 
for inbound tourist attractions, one DM may think it is "good", and the other 9 experts may think it is "medium". Then the evaluation information represented by HFLTS is $\{$ good, medium $\}$. Obviously, the result that does not consider the importance or weight of different linguistic terms may misrepresent the preferences of DMs. In order to avoid the above problems, Pang et al. (2016) introduced the probabilistic linguistic term set (PLTS) by adding the corresponding probability to the HFLTS, which reduces the information loss in the evaluation process and increases the DM's Express the applicability and accuracy of its linguistic assessment. Use PLTS to indicate that the result of the above example is $\{\operatorname{good}(0.1)$, medium $(0.9)\}$.PLTS can reflect the true weights of different attributes corresponding to the alternative and is suitable for group decision-making problems with distributed information (Wang et al. 2018a). Therefore, research on PLTS is necessary.

The rationality and flexibility of PLTS have attracted the attention of more and more scholars, and scholars have studied PLTS from different angles (Liao et al. 2019; Mi et al. 2020), including probabilistic linguistic vector item sets promote the application of multi-granular linguistic information (Zhai et al. 2016), MADM problem (Gou et al. 2016), the operation of PLTS, the distance measurement of PLTS(Lin et al. 2019; Lin and Xu 2018; Wang et al. 2018b; Zhang et al. 2020), the probability formula of PLTS (Bai et al. 2017; Feng et al. 2019; Xian et al. 2019; Yu et al. 2019a; Yu et al. 2019b), probabilistic linguistic preference relations (Gao et al. 2018; Song and Jun 2019; Tian et al. 2019; Zhang et al. 2018; Zhang et al. 2016), etc.

Actually, the process of determining attribute weights is equally important. The subjective weight given by the DMs is based on the relative importance of the standard (Lootsma 2010; Rodríguez et al. 2017), while the objective weight is obtained by evaluating the standard itself. Given the correlation between attributes, Diakoulaki et al. (1995) proposed an objective method of determining weight, which gives less weight to the more relevant attributes. In addition, some scholars combine subjective and objective weights to determine standard weights (Hafezalkotob and Hafezalkotob 2015). However, the above-mentioned weight determination techniques have two shortcomings 
(Wu et al. 2018): (1) It is difficult for them to express all the subjective preferences of DMs in a single linguistic term, and they cannot integrate the evaluations of all DMs without complete information. (2) There are relatively few studies on the determination of the objective weight of attributes in a probabilistic linguistic environment. In order to maintain objectivity, when studying the application of MAUT-PAMSSEM II in PLTS, this article uses the entropy method to determine the weight. And finally, we compare this new method with PROMETHEE, TOPSIS, and ARAS to show its applicability.

In summary, the focus of this article includes the following points:

(a) We provide a new decision-making method when the attribute weight is unknown, called the PL-MAUT-PAMSSEM II method. It merges the MAUT and PAMSSEM methods into two calculation stages in a probabilistic linguistic environment, which not only provides more method options for DMs but also improves the accuracy of evaluation considering the different importance of linguistic terms.

(b) The PL-MAUT-PAMSSEM II method combines PLTS and HPFS through the equivalent conversion functions and compares the alternatives in pairs according to the marginal utility score to obtain the ranking of the alternatives.

(c) Compare and analyze the method proposed in this article with existing methods.

(d) We apply the methods provided to solve the problem of inbound tourist destination selection.

The rest of this article is as follows: Section 2 reviews some basic knowledge of PLTS and HPFS. Section 3 introduces the MAUT method and PAMSSEM method respectively and includes the entire calculation process of PL-MAUT-PAMSSEM II. Section 4 introduces the entropy method and the whole process of determining attribute weights. Section 5 shows a case of inbound tourism destination selection and compares several methods to prove the effectiveness of the proposed PL-MAUT-PAMSSEM II method. Section 6 is the conclusion of this article.

\section{Preliminaries}


In this section, we will give some basic concepts and formulas of PLTS, HPFS and the equivalent transfer functions.

\subsection{The probabilistic linguistic term set}

Definition 1 (Pang et al. 2016) The probabilistic linguistic term set (PLTS) on LTS $L$ can be defined as:

$P L(p)=\left\{l^{(k)}\left(p^{(k)}\right) \mid l^{(k)} \in L, p^{(k)} \geq 0, k=1,2, \cdots, \# P L(p), \sum_{k=1}^{\# P L(p)} p^{(k)} \leq 1\right\}$,

Where $l^{(k)}\left(p^{(k)}\right)$ is made up of linguistic term $l^{(k)}$ and its associated probability $p^{(k)}$, and the cardinality of $P L(p)$ is $\# P L(p)$. If $k=p^{(k)}=1$, then the PLTS reduces to a linguistic term.

The size of PLTS can be compared by the following scoring function and variance function:

Definition 2 Let $\sigma\left(l^{(k)}\right)$ is the membership degree of the linguistic term $l^{(k)}$ in $\operatorname{PLTS} \operatorname{PL}(p)$, then the score function of $P L(p)$ is defined as:

$S(P L(p))=\sum_{k=1}^{\# P L(p)} \sigma\left(l^{(k)}\right) p^{(k)} / \sum_{k=1}^{\# P L(p)} p^{(k)}$

Definition 3 Suppose that $\sigma\left(l^{(k)}\right)$ is the membership degree of the linguistic term $l^{(k)}$ in PLTS $P L(p)$, and $S(P L(p))$ is the score value of the PLTS $P L(p)$.Then the variance function of $P L(p)$ is defined as:

$D(P L(p))=\left(\sum_{k=1}^{\# P L(p)}\left(\left(p^{(k)}\left(\sigma\left(l^{(k)}\right)-S(P L(p))\right)\right)^{2}\right)^{0.5}\right) / \sum_{k=1}^{\# P L(p)} p^{(k)}$

For two any PLTSs $P L_{1}(p)$ and $P L_{2}(p)$ with $S\left(P L_{1}(p)\right) \neq S\left(P L_{2}(p)\right)$,

(1) If $S\left(P L_{1}(p)\right)>S\left(P L_{2}(p)\right)$, then $P L_{1}(P)>P L_{2}(P)$;

(2) If $S\left(P L_{1}(p)\right)<S\left(P L_{2}(p)\right)$, then $P L_{1}(P)<P L_{2}(P)$;

(3) If $S\left(P L_{1}(p)\right)=S\left(P L_{2}(p)\right)$, then

(i) If $D\left(P L_{1}(P)\right)>D\left(P L_{2}(P)\right)$, then $P L_{1}(P)<P L_{2}(P)$

(ii) If $D\left(P L_{1}(P)\right)<D\left(P L_{2}(P)\right)$, then $P L_{1}(P)>P L_{2}(P)$

(iii) If $D\left(P L_{1}(P)\right)=D\left(P L_{2}(P)\right)$, then $P L_{1}(P) \approx P L_{2}(P)$ 


\subsection{The hesitant probabilistic fuzzy set}

Definition 4 (Torra 2010; Torra and Narukawa 2009) Let $X$ be a reference set. Accordingly, an HFS A on $X$ is defined in terms of a function $h_{A}(x)$ that when applied to $X$ returns a finite subset of $[0,1]$ :

$A=\left\{<x, h_{A}(x)>\mid x \in X\right\}$,

Where $h_{A}(x)$ is a set of multiple different values on $[0,1]$, which represents the membership degree of the element $x$ in $X$ on $A$, and $h_{A}(x)$ represents HFE (Xia and Xu 2011).

Example 1 Let $X=\left\{x_{1}, x_{2}\right\}$ be a reference set, and $h_{A}\left(x_{1}\right)=\{0.1,0.3\}$ and $h_{A}\left(x_{2}\right)=\{0.2,0.3,0.5\}$ be two HFEs of $x_{i}=(i=1,2)$ to a set $A$. Then, $A$ can be expressed as an HFS as follows:

$A=\left\{\left\langle x_{1},\{0.1,0.3\}\right\rangle,\left\langle x_{2},\{0.2,0.3,0.5\}\right\rangle\right\}$

Definition 5 (Xu and Zhou 2016) Let $X$ be a reference set, then an HPFS $A_{p}$ on $X$ can be expressed by as:

$A_{p}=\left\{<x, h\left(p_{x}\right)>\mid x \in X\right\}$

Where $h\left(p_{x}\right)$ is a set of multiple different values on $[0,1]$, which described by a probability distribution $p_{x}$. Among them, $p_{x}$ represents the membership degree of the elements $x$ on the set $X$ on $A_{p} \cdot h\left(p_{x}\right)$ can be expressed as an HPFE as follows:

$h\left(p_{x}\right)=\left\{\gamma_{i}\left(p_{i}\right) \mid i=1,2, \cdots, k\right\}$

Among them, $p_{i}$ represents probability of the possible value $\gamma_{i}$, and it satisfies $\sum_{i=1}^{k} p_{i}=1, k$ represents the number of $\gamma_{i}\left(p_{i}\right)$ in $h\left(p_{x}\right)$

Example 2 Let $X=\left\{x_{1}, x_{2}\right\}$ be a reference set, and $h\left(p_{1}\right)=\{0.1(0.6), 0.2(0.4)\}$ and $h\left(p_{2}\right)=\{0.6(0.2), 0.2(0.8)\}$ be two HPFS as follows:

$E_{P}=\left\{\left\langle x_{1},\{0.1(0.6), 0.2(0.4)\}\right\rangle,\left\langle x_{2},\{0.6(0.2), 0.2(0.8)\}\right\rangle\right\}$.

The size of HPFS can be compared by the following scoring function and variance function:

Definition 6 (Zhang et al. 2017) Let $h\left(p_{x}\right)=\left\{\gamma_{i}\left(p_{i}\right) \mid i=1,2, \cdots, k\right\}$ be an HPFE. Its expected mean can be defined as follows: 
$E\left(h\left(p_{x}\right)\right)=\sum_{i=1}^{k} \gamma_{i} p_{i}$

Based on the expected mean, the variance of an HPFE can be defined as follows.

Definition 7 Let $h\left(p_{x}\right)=\left\{\gamma_{i}\left(p_{i}\right) \mid i=1,2, \cdots, k\right\}$ be an HPFE. Its variance can be defined as follows:

$D\left(h\left(p_{x}\right)\right)=\sum_{i=1}^{k}\left(\gamma_{i}-E\left(h\left(p_{x}\right)\right)\right)^{2} p_{i} ;$

Definition 8 (Xu and Zhou 2016) Let $\bar{h}_{i}(i=1,2, \cdots, I)$ be a collection of HPFS, $\omega=\left(\omega_{1}, \omega_{2}, \cdots, \omega_{i}\right)^{T}$ be the

weight vector of $\bar{h}_{i}$ with $\omega_{i} \in[0,1]$ and $\sum_{i=1}^{I} \omega_{i}=1, p_{i}$ be the probability of $\gamma_{i}$ in the HPFE $\bar{h}_{i}, \gamma_{\sigma(i)}$

be the $i$ th largest of $\bar{h}_{i}, p_{\sigma(i)}$ be the probability of $\gamma_{\sigma(i)}$ in the HPFE $\bar{h}_{i}$, and $\omega_{\sigma(i)}$ be the $i$ th largest of $\omega$, then the aggregation operator can be defined as follows:

$$
\operatorname{HPFOWA}\left(\bar{h}_{1}, \bar{h}_{2}, \cdots, \overline{h_{i}}\right)=\bigoplus_{i=1}^{I}\left(\omega_{i} \bar{h}_{\sigma(i)}\right) \bigcup_{\gamma_{\sigma(1)} \bar{h}_{\sigma(1)}, \gamma_{\sigma(2)} \in \bar{h}_{\sigma(2)}, \cdots, \gamma_{\sigma(i)} \in \bar{h}_{\sigma(i)}}\left\{1-\prod_{i=1}^{I}\left(1-\gamma_{\sigma(i)}\right)^{\omega_{i}} p_{\sigma(1)} p_{\sigma(2)} \cdots p_{\sigma(i)}\right\}
$$

\subsection{The equivalent transformation functions}

Definition 9 (Gou and $\mathrm{Xu}$ 2016; Zhang and Qi 2013) Let $h_{L}=\left\{l_{t} \mid t \in[-\delta, \delta]\right\}$ be a HFLE on LTS $L=\left\{l_{t} \mid t=-\delta, \cdots,-1,0,1, \cdots, \delta\right\}$, and $H=\{\varepsilon \mid \varepsilon \in[0,1]\}$ be a HFS. Then the linguistic variable $l_{t}$ and membership degree $\varepsilon$ can be transformed into each other by the function $\sigma$ and $\sigma^{-1}$ given as:

$$
\begin{aligned}
& \sigma:[-\delta, \delta] \rightarrow[0,1], \\
& \sigma\left(h_{L}\right)=\left\{\sigma\left(l_{t}\right)=\varepsilon=\frac{t}{2 \delta}+\frac{1}{2} \mid t \in[-\delta, \delta]\right\}=h_{\varepsilon} ; \\
& \sigma^{-1}:[0,1] \rightarrow[-\delta, \delta], \\
& \sigma^{-1}\left(h_{\varepsilon}\right)=\left\{\sigma^{-1}(\varepsilon)=l_{t}=l(2 \varepsilon-1) \delta \mid \varepsilon=[0,1]\right\}=h_{L} .
\end{aligned}
$$

\subsection{Some operations of PLTSs}

Definition 10 (Yue et al. 2020) Let $P L(p), P L_{1}(p)$ and $P L_{2}(p)$ be three adjusted PLTSs on LTS $L$, and $\lambda$ be a positive real number. Then

$$
\begin{aligned}
& \text { (1) } P L_{1}(p) \oplus P L_{2}(p)=\bigcup_{\xi_{1}^{(k)} \in \sigma\left(P L_{1}\right), \xi_{2}^{(k)} \in \sigma\left(P L_{2}\right)}\left\{\sigma^{-1}\left(\xi_{1}^{(k)}+\xi_{2}^{(k)}-\xi_{1}^{(k)} \xi_{2}^{(k)}\right)\left(p^{(k)}\right)\right\}, k=1,2, \cdots, \# P L_{1}(p)=\# P L_{1}(p) ; \\
& \text { (2) } P L_{1}(p) \otimes P L_{2}(p)=\bigcup_{\xi_{1}^{(k)} \in \sigma\left(P L_{1}\right), \xi_{2}^{(k)} \in \sigma\left(P L_{2}\right)}\left\{\sigma^{-1}\left(\xi_{1}^{(k)} \xi_{2}^{(k)}\right)\left(p^{(k)}\right)\right\}, k=1,2, \cdots, \# P L_{1}(p)=\# P L_{1}(p) ; \\
& \text { (3) } P L_{1}(p) \ominus P L_{2}(p)=\bigcup_{\xi_{1}^{(k)} \in \sigma\left(P L_{1}\right), \xi_{2}^{(k)} \in \sigma\left(P L_{2}\right)}\left\{\sigma^{-1}(\Pi)\left(p^{(k)}\right)\right\} ;
\end{aligned}
$$


where

$\Pi=\left\{\begin{array}{cc}\frac{\xi_{1}^{(k)}-\xi_{2}^{(k)}}{1-\xi_{2}^{(k)}} & \text { if } \xi_{1}^{(k)} \geq \xi_{2}^{(k)} \text { and } \xi_{2}^{(k)} \neq 1 \\ 0 & \text { if otherwise }\end{array}\right.$

and $k=1,2, \cdots, \# P L_{1}(p)=\# P L_{1}(p)$;

(4) $P L_{1}(p) \oslash P L_{2}(p)=\bigcup_{\xi_{1}^{(k)} \in \sigma\left(P L_{1}\right), \xi_{2}^{(k)} \in \sigma\left(P L_{2}\right)}\left\{\sigma^{-1}(\Pi)\left(p^{(k)}\right)\right\}$

where

$\Pi=\left\{\begin{array}{cc}\frac{\xi_{1}^{(k)}}{\xi_{2}^{(k)}} & \text { if } \xi_{1}^{(k)} \leq \xi_{2}^{(k)} \text { and } \xi_{2}^{(k)} \neq 1 \\ 0 & \text { if otherwise },\end{array}\right.$

and $k=1,2, \cdots, \# P L_{1}(p)=\# P L_{1}(p)$.

\section{Combinatorial of the MAUT and PAMSSEM II methods}

\subsection{The PAMSSEM method}

The PAMSSEM methods were introduced by Martel (1998) and is applied to cloud resource integration management (Yazir et al. 2012). The PAMSSEM method includes stages I and II. The first stage gives a partial ranking of the alternatives by calculating entering and leaving flow, and the second stage gives a complete ranking of the alternatives by calculating net flow. The purpose of PAMSSEM is to establish a preference relationship (Bélanger et al. 2000a; Bélanger et al. 2000b; Bélanger and Martel 2012). After DMS are given a decision matrix, the indifference threshold parameters(q), the preference threshold(p), and the veto threshold(v), the alternatives are ranked by establishing a local preference model (Alinezhad and Khalili 2019).

The PROMETHEE method is proposed to deal with the incomparable situation when comparing targets (Figueira et al. 2005). This method is simple, clear, and stable (Brans et al. 1986). As an improvement of this method, PAMSSEM also has the above advantages. In addition, the advantages of the PAMSSEM method also 
include the ability to handle missing evaluations, the discrimination threshold allows $A_{i}$ better than $A_{i}$, while $A_{i}$ is better than $A_{i}$ and the rejection threshold can prevent $A_{i}$ from being better than $A_{i}$. Among them, when the threshold value is biased, it may have an impact on the ranking results, but this does not conflict with the stability of the method. Changes in parameters will inevitably lead to changes in rankings, which just shows that the method considers the particularity of each scheme.

In mathematics, the probability density function of a continuous random variable is a function that describes the probability that the output value of this random variable is near a certain value point. The probability that the value of a random variable falls within a certain region is integral to the probability density function in this region. The decision matrix given by DMS represents the distribution of random variables (or statistical variables) $X_{i j}$ with probability density or quality function $f_{i j}\left(x_{i j}\right)$. A clear assessment can be seen as the degradation of the distribution, the distribution is reduced to only a point $\left(\left(p\left(X_{i j}=e_{i j}\right)=1\right)\right)$, and the probability distribution function is as follows (Guitouni et al. 1999):

$$
\int_{-\infty}^{\infty} f_{i j}\left(x_{i j}\right) d x_{i j}=1
$$

\subsection{The MAUT method}

The Muti-Attribute Utility Theory (MAUT) method was introduced by Keeney and Raiffa in 1976 (Emovon et al. 2016; Figueira et al. 2005). The hypothesis of this method is that each DM tries to consciously or implicitly optimize an aggregate function that integrates the opinions of all DMs. This function may not be known before the start of the decision, so DMs need to construct it first (Nemery and Ishizaka 2013; Wallenius et al. 2008). The utility function in economics is a function used to express the quantitative relationship between the utility obtained by consumers in consumption and the combination of commodities consumed, to measure the degree of satisfaction that consumers obtain from the consumption of a given combination of commodities. Compared with the previous method, this method is more convenient in the operation of solving MAGDM problems, which is one of its 
advantages. This method gives DMs more initiative, and makes the evaluation results more real and accurate, and can better reflect the wishes of DMs.

\subsection{The PL-MAUT-PAMSSEM II method}

Based on the advantages of the MAUT method and the PAMSSEM method proposed above, we combine the two methods and apply them to PLTSs. PAMSSEM is a method of ranking programs based on preference. Based on the 2004-2019 inbound tourism data provided by the official website and statistical yearbook, we invite multiple DMs to evaluate multiple popular inbound tourist destinations and let the DMs set specific thresholds $\mathrm{p}$, $\mathrm{q}$, and $\mathrm{v}$. Through the threshold $\mathrm{p}, \mathrm{q}, \mathrm{v}$ to remove some attribute values, the attribute value closest to the threshold $\mathrm{p}$ can reflect the preference of DMs. MAUT is a method of ranking alternatives based on the utility function. A utility function is also a tool that reflects the preferences or intentions of decision makers. Therefore, this article combines the two methods to optimize the decision-making process, make the decision-making results more real and accurate, and provide DMs with better travel plans.

The decision-making processes based on PL-MAUT-PAMSSEM II method are as follows:

For a MAGDM problem in PLTS, let $A=\left\{A_{1}, A_{2}, \cdots, A_{m}\right\}$ be a finite set of alternatives, $C=\left\{C_{1}, C_{2}, \cdots, C_{n}\right\}$ be the set of attributes and $\omega=\left(\omega_{1}, \omega_{2}, \cdots, \omega_{n}\right)^{T}$ be the weight vector of attributes $C_{j}(j=1,2, \cdots, n)$, with $\omega_{j} \in[0,1], j=1,2, \cdots, n$ and $\sum_{j=1}^{n} \omega_{j}=1$.Suppose that $R_{y}=\left[L S(p)_{i j}\right]_{m \times n}$ is the decision matrix for year $\mathrm{y}$, where $L S(p)_{i j}=\left\{L S_{i j}^{(t)}\left(p_{i j}^{(t)}\right) \mid t=1,2, \cdots, \# L S(p)_{i j}\right\} \quad$ is a PLTS, which is an evaluation value of alternative $A_{i}$ about attribute $C_{j}$. Then the goal is to rank the alternatives.

Step1 Receive information from decision makers and form the PL decision-making matrixes $R_{y}=\left[L S(P)_{i j}\right]_{m \times n}, \quad y=1,2, \cdots, Y$.

Step2 Calculate the decision matrix $R=\left[L S(P)_{i j}\right]_{m \times n}$ after aggregation by formulas (8) and (9). 
$R=\left[\begin{array}{ccccc}L S(p)_{11} & \cdots & L S(p)_{1 j} & \cdots & L S(p)_{1 n} \\ \vdots & \ddots & \vdots & \ddots & \vdots \\ L S(p)_{i 1} & \cdots & L S(p)_{i j} & \cdots & L S(p)_{i n} \\ \vdots & \ddots & \vdots & \ddots & \vdots \\ L S(p)_{m 1} & \cdots & L S(p)_{m j} & \cdots & L S(p)_{m n}\end{array}\right]_{m \times n} \quad ; i=1,2, \cdots, m, j=1,2, \cdots, n$

Step3 Normalized the decision matrix $R^{*}=\left[L S(P)^{*}{ }_{i j}\right]_{m \times n}$.

Step4 Use formula (1) to get the score matrix of the alternatives under different attributes $S$ :

$$
S=\left[\begin{array}{ccccc}
s\left(L S(p)_{11}^{*}\right) & \cdots & s\left(L S(p)_{1 j}^{*}\right) & \cdots & s\left(L S(p)_{1 n}^{*}\right) \\
\vdots & \ddots & \vdots & \ddots & \vdots \\
s\left(L S(p)_{i 1}^{*}\right) & \cdots & s\left(L S(p)_{i j}^{*}\right) & \cdots & s\left(L S(p)_{i n}^{*}\right) \\
\vdots & \ddots & \vdots & \ddots & \vdots \\
S\left(L S(p)_{m 1}^{*}\right) & \cdots & s\left(L S(p)_{m j}^{*}\right) & \cdots & s\left(L S(p)_{m n}^{*}\right)
\end{array}\right]_{m \times n} ; i=1,2, \cdots, m, j=1,2, \cdots, n
$$

In order to calculate the utility function, the DMs needs to first standardize the score value matrix to readjust the original performance between 0 and 1 . The rescaling or normalization step is usually based on the minimum and maximum performance of the alternatives on each attribute:

$s_{j}^{\prime}\left(a_{i}\right)=\frac{s_{j}\left(a_{i}\right)-\min \left(s_{j}\right)}{\max \left(s_{j}\right)-\min \left(s_{j}\right)}$

When maximizing the attribute, or

$s_{j}^{\prime}\left(a_{i}\right)=1+\left(\frac{\min \left(s_{j}\right)-s_{j}\left(a_{i}\right)}{\max \left(s_{j}\right)-\min \left(s_{j}\right)}\right)$

When minimizing the attribute.

Step5 Calculate the weight of each attribute $\omega_{j}$ by formula (28)-(32).

Step6 Calculate the marginal utility score as follows:

$u_{i j}=\frac{e^{\left(s^{\prime}\left(L S(p)_{i j}^{*}\right)\right)^{2}}-1}{1.71} ; \quad i=1,2, \cdots, m, j=1,2, \cdots, n$

Step7 Calculate the local outranking index $\delta_{j}\left(A_{i}, A_{i^{\prime}}\right)$ by the following expression: 
$\delta_{j}\left(A_{i}, A_{i^{\prime}}\right)=\sum_{A_{i^{\prime}}}\left(\sum_{A_{i}} \bar{\delta}_{j}\left(A_{i}, A_{i^{\prime}}\right) \cdot f_{j}\left(A_{i}\right)\right) \cdot f_{j}\left(A_{i^{\prime}}\right) ; \quad i, i^{\prime} \in\{1,2, \cdots, m\}$

$f_{j}\left(A_{i^{\prime}}\right)$ and $f_{j}\left(A_{i}\right)$ are respectively the probability density functions (discrete) which are assumed to be equal one. $\bar{\delta}_{j}\left(A_{i}, A_{i^{\prime}}\right)$ is index computed according to the following formula:

$$
\bar{\delta}_{j}\left(A_{i}, A_{i^{\prime}}\right)=\left\{\begin{array}{cc}
0 & \text { if } \Delta_{j} \leq-p_{j} \\
\frac{\Delta_{j}+p_{j}}{p_{i}-q_{j}} & \text { if }-p_{j}<\Delta_{j}-q_{j} ; \quad p_{j} \geq q_{j} \geq 0 \\
1 & \text { if } \Delta_{j} \geq-q_{j}
\end{array}\right.
$$

Where $\Delta_{j}=u_{i j}-u_{i^{\prime} j}$, which indicates the difference between the level, and threshold $\mathrm{q}, \mathrm{p}$ is the values determined by the DMs for each attribute. When the number of ordinal levels is greater than 3, after modification, we can get the same equivalent function as ordinal evaluation local outranking index:

$$
\bar{\delta}_{j}\left(A_{i}, A_{i^{\prime}}\right)=\left\{\begin{array}{lc}
0 & \text { if } \Delta_{j} \leq-1 \\
\frac{1}{2} & \text { if }-1<\Delta_{j}<0 ; \quad i, i^{\prime} \in\{1,2, \cdots, m\} \\
1 & \text { if } \Delta_{j} \geq 0
\end{array}\right.
$$

Step8 Calculate the concordance index $C\left(A_{i}, A_{i^{\prime}}\right)$ for each pair of alternatives as follows:

$$
C\left(A_{i}, A_{i^{\prime}}\right)=\sum_{j=1}^{n} \delta_{j}\left(A_{i}, A_{i^{\prime}}\right) \cdot \omega_{j} ; \quad i, i^{\prime} \in\{1,2, \cdots, m\}
$$

Where $\omega_{j}$ represent the weight of each attribute and $\sum_{j=1}^{n} \omega_{j}=1$.

Step9 Calculate the local discordance index $D\left(A_{i}, A_{i^{\prime}}\right)$ as follows:

$$
D_{j}\left(A_{i}, A_{i^{\prime}}\right)=\sum_{A_{i}}\left(\sum_{A_{i^{\prime}}} \bar{D}_{j}\left(A_{i}, A_{i^{\prime}}\right) \cdot f_{j}\left(A_{i^{\prime}}\right)\right) \cdot f_{j}\left(A_{i}\right)
$$

$f_{j}\left(A_{i^{\prime}}\right)$ and $f_{j}\left(A_{i}\right)$ are respectively the probability density functions (discrete) which are assumed to be equal one. $\bar{D}_{j}\left(A_{i}, A_{i^{\prime}}\right)$ is index computed according to the following formula: 


$$
\bar{D}_{j}\left(A_{i}, A_{i^{\prime}}\right)=\left\{\begin{array}{cc}
0 & \text { if } \Delta_{j} \leq-v_{j} \\
-\frac{\left(\Delta_{j}+p_{j}\right)}{v_{j}-p_{j}} & \text { if }-v_{j}<\Delta_{j}<-p_{j} ; \quad v_{j}>p_{j} \\
1 & \text { if } \Delta_{j} \geq-p_{j}
\end{array}\right.
$$

Where the threshold $\mathrm{v}$ is determined by DMs. In addition, $\bar{D}_{j}\left(A_{i}, A_{i^{\prime}}\right)$ for the cardinal attributes is determined by following formula:

$$
\bar{D}_{j}\left(A_{i}, A_{i^{\prime}}\right)=\left\{\begin{array}{cl}
\min \left[1,-\xi\left(\omega_{j}\right) \cdot\left(\Delta_{j}+\frac{\alpha_{j}+1}{2}\right)\right] & \text { if } \Delta_{j}<-\left(\frac{\alpha_{j}+1}{2}\right) \\
0 & \text { if } \Delta_{j} \geq-\left(\frac{\alpha_{j}+1}{2}\right)
\end{array}\right.
$$

Where $\alpha_{j}$ is the number of measurement scale levels of the $\mathrm{j}$-th attribute $\left(\alpha_{j}>3\right)$ and $\xi\left(\omega_{j}\right)$ is a non-decreasing function of the relative importance of the $\mathrm{j}$-th criterion. This function can be calculated by following formula:

$\xi\left(\omega_{j}\right)=0.2\left(1+\frac{\omega_{j}}{2}\right) ; \quad j=1,2, \cdots, n$

Step10 Calculate the outranking degree $\varphi\left(A_{i}, A_{i^{\prime}}\right)$ as follows:

$$
\varphi\left(A_{i}, A_{i^{\prime}}\right)=C\left(A_{i}, A_{i^{\prime}}\right) \cdot \prod_{j=1}^{n}\left[1-\bar{D}^{3}\left(A_{i}, A_{i^{\prime}}\right)\right] ; \quad 0 \leq \varphi\left(A_{i}, A_{i^{\prime}}\right) \leq 1
$$

Step11 Calculate the entering and leaving flows of each alternative:

$$
\begin{gathered}
\varphi^{+}\left(A_{i}\right)=\sum_{A_{i} \in A} \varphi\left(A_{i}, A_{i^{\prime}}\right) ; \quad i, i^{\prime} \in\{1,2, \cdots, m\} \\
\varphi^{-}\left(A_{i}\right)=\sum_{A_{i} \in A} \varphi\left(A_{i}, A_{i^{\prime}}\right) ; \quad i, i^{\prime} \in\{1,2, \cdots, m\}
\end{gathered}
$$

Step12 Calculate the net flow of each alternative:

$$
\varphi\left(A_{i}\right)=\varphi^{+}\left(A_{i}\right)-\varphi^{-}\left(A_{i}\right)
$$

Step13 Rank all alternatives according to the value of $\varphi\left(A_{i}\right)$.

\section{The Entropy method}


Definition 11 (He et al. 2016; Wang et al. 2015; Xu et al. 2021; Yang and Cui 2011; Zou et al. 2006). The entropy method is an objective weighting method, which determines the weight of the attribute according to the information provided by the observation value of each attribute. This article combines the weights obtained by objective calculations and the decision-making information obtained by subjective evaluation to give the ranking of some popular inbound tourism areas. According to $m$ alternatives and $\mathrm{n}$ attributes, the DMs give the decision matrix, and the steps of the entropy method to determine the weight are as follows:

Step1 Calculate the proportion of the i-th alternative under the j-th attribute as follows:

$$
q_{i j}=\frac{s^{\prime}\left(L S(p)_{i j}^{*}\right)}{\sum_{i=1}^{m} s^{\prime}\left(L S(p)_{i j}^{*}\right)}(i=1,2, \cdots, m, j=1,2, \cdots, n), \sum_{i=1}^{n} q_{i j}=1
$$

Step2 Calculate the entropy value of the $\mathrm{j}$-th index by following formula:

$e_{j}=-k \times \sum_{i=1}^{n} q_{i j} \ln \left(q_{i j}\right) ; \quad i=1,2, \cdots, m, j=1,2, \cdots, n$

Where $k>0$, ln is the natural logarithm, and $e_{i j} \geq 0$. Generally let $k=\frac{1}{\ln m}$, then $0 \leq e \leq 1$. When $q_{i j}=0$, define $\lim _{q_{i j} \rightarrow 0}\left(q_{i j} \ln q_{i j}\right)=0$.

Step3 Calculate the difference coefficient of the $\mathrm{j}$-th index by following formula:

$$
g_{j}=1-e_{j} ; \quad j=1,2, \cdots, n
$$

Step4 Calculate the weights by following formula:

$$
\omega_{j}=\frac{g_{j}}{\sum_{j=1}^{n} g_{j}} ; j=1,2, \cdots, n
$$

Step5 The final weight vector $\Omega$ is:

$$
\Omega=\left(\omega_{1}, \omega_{2}, \omega_{3}, \omega_{4}, \omega_{5}\right)
$$

\section{Case Study: Choice of tourist attractions}

In this section, we will use the PL-MAUT-PAMSSEM II method proposed above to rank the alternatives, so as to 
provide decision-makers with selection reference.

\subsection{Case presentation}

After a long period of continuous research on the influencing factors of regional inbound tourism development, a relatively complete attribute index system has been formed. This article selects economic level, openness, hotel supply, transportation facilities, and tourism resources as the attribute indicators for the evaluation of inbound tourism competitiveness (Wu et al. 2020), The number of inbound tourists is selected as a reference index to test the validity and accuracy of the competitiveness evaluation. Among them, the number of inbound tourists is measured by the number of inbound overnight tourists, the economic level is measured by GDP, and the degree of openness is measured by the volume of imports and exports. At the same time, the fluctuation of domestic price and consumption level needs to be eliminated. The direct economic effect of the exchange rate change of the RMB against the US dollar and the fluctuation of the domestic prices and consumption level need to eliminate the direct economic effect of the import and export volume, so as to ensure the annual volume comparability of the GDP and the import and export volume; hotel supply passed comprehensive measurement of one-star, two-star, three-star, four-star and five-star hotels (houses); transportation facilities are comprehensively measured by inland river network density, highway network density, railway network density, and air transportation employment density; Tourism resources are comprehensively measured through A-level scenic spots, historical and cultural cities, world natural heritage and world cultural heritage.

The data required for this article comes from the official websites of the National Bureau of Statistics of the People's Republic of China, the People's Bank of China, the Ministry of Culture and Tourism of the People's Republic of China, 2001-2019 "Inbound Tourist Sampling Survey Data", "China Tourism Statistical Yearbook", "Fujian Statistical Yearbook" "Xinjiang Statistical Yearbook" and other provincial-level regional statistical yearbooks. According to relevant statistics in 2007, 2011, 2015 and 2019(these four years of data have the same 
importance), 10 DMs were invited to $\mathrm{A}_{1}$ (Guangdong), $\mathrm{A}_{2}$ (Yunnan), $\mathrm{A}_{3}$ (Shaanxi), $\mathrm{A}_{4}$ (Sichuan), $\mathrm{A}_{5}$ (Inner Mongolia) five inbound tourist hotspots in $\mathrm{C}_{1}$ (economic level), $\mathrm{C}_{2}$ (openness), $\mathrm{C}_{3}$ (hotel supply), $\mathrm{C}_{4}$ (transportation facilities), $\mathrm{C}_{5}$ (tourism resources) scores under five standards to obtain decision-making information. The weight vector of criteria is $\Omega=\left(\omega_{1}, \omega_{2}, \omega_{3}, \omega_{4}, \omega_{5}\right)$, which can calculated by formula (35), where $0 \leq \omega_{j} \leq 1$ and $\sum_{j=1}^{5} \omega_{j}=1$ and LTS

$L=\left\{l_{-3}=\right.$ terrible,$l_{-2}=$ very bad $, l_{-1}=\mathrm{bad}, l_{0}=$ medium $, l_{1}=\operatorname{good}, \quad l_{2}=$ very good, $l_{3}=$ fantastic $\}$. In order to obtain the decision matrixes, we collected the scores of 10 DMs in 2007, 2011, 2015 and 2019 and then convert them into PLTS through linguistic scale. Then, we listed the final assessments of these tourist attractions in Table 1-4, and form the probabilistic linguistic decision matrixes $\left(P L_{i j}(p)\right)_{5 \times 5}$.

\subsection{The Decision Steps}

Step 1 Split the matrix, take attribute $C_{1}$ as an example, we can get Table 5.

Step 2 Calculate the hesitant probabilistic fuzzy decision matrix by formula (9), take attribute $C_{1}$ as an example, we can get Table 6.

Step 3 Calculate the aggregated hesitant probabilistic fuzzy decision matrix by formula (8), and we can get Table 7.

Step 4 Calculate the probabilistic linguistic decision matrix by formula (9), we can get Table 8 .

Step 5 Using formula (1), we can obtain the score as follows:

$S=\left[\begin{array}{ccccc}2.896 & 3 & 3 & 2.565 & 2.983 \\ 2.832 & 2.903 & 3 & 3 & 2.765 \\ 3 & 2.486 & 2.318 & 2.900 & 2.707 \\ 3 & 2.779 & 2.792 & 2.516 & 2.863 \\ 1.020 & 0.542 & 2.336 & 2.395 & 2.224\end{array}\right]$

Step 6 Readjust the score matrix as follows: 


$$
S^{\prime}=\left[\begin{array}{ccccc}
0.948 & 1 & 1 & 0.281 & 1 \\
0.915 & 0.961 & 1 & 1 & 0.713 \\
1 & 0.791 & 0 & 0.835 & 0.636 \\
1 & 0.910 & 0.695 & 0.200 & 0.842 \\
0 & 0 & 0.026 & 0 & 0
\end{array}\right]
$$

Step 7 Using formula (28)-(32), we can obtain the attribute weight as follows:

$$
\omega=(0.142,0.144,0.304,0.260,0.150)^{T}
$$

Step 8 Calculate the marginal utility score by formula (15), and we can get:

$$
u_{i j}=\left[\begin{array}{ccccc}
0.852 & 1.005 & 1.005 & 0.048 & 1.005 \\
0.766 & 0.888 & 1.005 & 1.005 & 0.387 \\
1.005 & 0.508 & 0 & 0.590 & 0.292 \\
1.005 & 0.754 & 0.363 & 0.024 & 0.603 \\
0 & 0 & 0 & 0 & 0
\end{array}\right]
$$

Step 9 Calculate the concordance index $C\left(A_{i}, A_{i}\right)$ by formula (16)-(19), and Table 9 indicates the parameters q, $\mathrm{p}$,

and $\mathrm{v}$ of each attribute, and we can get:

$$
C\left(A_{i}, A_{i^{\prime}}\right)=\left[\begin{array}{ccccc}
/ & 1 & 1 & 1 & 1 \\
1 & / & 1 & 1 & 1 \\
0.856 & 0.856 & / & 1 & 1 \\
1 & 1 & 1 & / & 1 \\
0.784 & 0.856 & 1 & 1 & /
\end{array}\right]
$$

Step 10 Calculate the local discordance index $D\left(A_{i}, A_{i}\right)$ by formula (20)-(23), and we can get:

$$
D\left(A_{i}, A_{i^{\prime}}\right)=\left[\begin{array}{ccccc}
/ & 0 & 0 & 0 & 0 \\
0 & / & 0 & 0 & 0 \\
0 & 0 & / & 0 & 0 \\
0 & 0 & 0 & / & 0 \\
0 & 0 & 0 & 0 & /
\end{array}\right]
$$

Step 11 Calculate the outranking degree $\varphi\left(A_{i}, A_{i^{\prime}}\right)$ by formula (24), and we can get:

$$
\varphi\left(A_{i}, A_{i^{\prime}}\right)=\left[\begin{array}{ccccc}
/ & 1 & 1 & 1 & 1 \\
1 & / & 1 & 1 & 1 \\
0.856 & 0.856 & / & 1 & 1 \\
1 & 1 & 1 & / & 1 \\
0.784 & 0.856 & 1 & 1 & /
\end{array}\right]
$$


Step 12 Calculate the entering and leaving flows of each $A_{i}(i=1,2,3,4,5)$ by formula (25)-(26), we can get:

$$
\begin{aligned}
& \varphi^{+}\left(A_{i}\right)=(4,4,3.712,4,3.640) \\
& \varphi^{-}\left(A_{i}\right)=(3.640,3.712,4,4,4)
\end{aligned}
$$

Step 13 Calculate the net flows of each $A_{i}(i=1,2,3,4,5)$ by formula (27), and we can get:

$$
\varphi\left(A_{i}\right)=(0.360,0.288,-0.288,0,-0.360)
$$

Step 14 Rank the alternatives.

\begin{tabular}{|c|c|c|c|c|c|}
\hline & $\mathrm{C}_{1}$ & $\mathrm{C}_{2}$ & $\mathrm{C}_{3}$ & $\mathrm{C}_{4}$ & $\mathrm{C}_{5}$ \\
\hline $\mathrm{A}_{1}$ & $\left\{1_{2}(0.5), 1_{3}(0.5)\right\}$ & $\left\{1_{3}(1)\right\}$ & $\left\{1_{2}(0.2), 1_{3}(0.8)\right\}$ & $\left\{1_{1}(0.2), 1_{2}(0.8)\right\}$ & $\left\{\mathrm{l}_{2}(0.2), \mathrm{l}_{3}(0.8)\right\}$ \\
\hline $\mathrm{A}_{2}$ & $\left\{\mathrm{l}_{2}(0.9), \mathrm{l}_{3}(0.1)\right\}$ & $\left\{\mathrm{l}_{2}(0.8), \mathrm{l}_{3}(0.2)\right\}$ & $\left\{\mathrm{l}_{2}(0.5), \mathrm{l}_{3}(0.5)\right\}$ & $\left\{1_{3}(1)\right\}$ & $\left\{1_{1}(0.5), 1_{2}(0.5)\right\}$ \\
\hline $\mathrm{A}_{3}$ & $\left\{1_{0}(0.2), 1_{1}(0.8)\right\}$ & $\left\{1_{1}(1)\right\}$ & $\left\{\mathrm{l}_{0}(0.2), \mathrm{l}_{1}(0.8)\right\}$ & $\left\{1_{2}(0.5), 1_{3}(0.5)\right\}$ & $\left\{\mathrm{l}_{2}(0.8), \mathrm{l}_{3}(0.2)\right\}$ \\
\hline $\mathrm{A}_{4}$ & $\left\{1_{3}(1)\right\}$ & $\left\{1_{1}(0.7), 1_{2}(0.3)\right\}$ & $\left\{1_{1}(0.6), 1_{2}(0.4)\right\}$ & $\left\{1_{1}(0.7), 1_{2}(0.3)\right\}$ & $\left\{1_{1}(0.3), 1_{2}(0.7)\right\}$ \\
\hline $\mathrm{A}_{5}$ & $\left\{1_{-2}(0.3), 1_{-1}(0.7)\right\}$ & $\left\{1_{-1}(0.2), 1_{0}(0.8)\right\}$ & $\left\{1_{-2}(0.1), 1_{0}(0.9)\right\}$ & $\left\{1_{0}(0.9), 1_{1}(0.1)\right\}$ & $\left\{1_{1}(1)\right\}$ \\
\hline
\end{tabular}

According to the value of $\varphi\left(A_{i}\right)$, the ranking is $A_{1} \succ A_{2} \succ A_{4} \succ A_{3} \succ A_{5}$

Table 1 The probabilistic linguistic decision matrix for the year 2007.

Table 2 The probabilistic linguistic decision matrix for the year 2011.

\begin{tabular}{|c|c|c|c|c|c|}
\hline & $\mathrm{C}_{1}$ & $\mathrm{C}_{2}$ & $\mathrm{C}_{3}$ & $\mathrm{C}_{4}$ & $\mathrm{C}_{5}$ \\
\hline $\mathrm{A}_{1}$ & $\left\{l_{2}(1)\right\}$ & $\left\{1_{2}(0.3), 1_{3}(0.7)\right\}$ & $\left\{l_{2}(0.4), l_{3}(0.6)\right\}$ & $\left\{1_{1}(0.6), 1_{2}(0.4)\right\}$ & $\left\{l_{2}(0.5), l_{3}(0.5)\right\}$ \\
\hline $\mathrm{A}_{2}$ & $\left\{1_{1}(0.5), 1_{2}(0.5)\right\}$ & $\left\{\mathrm{l}_{2}(0.9), \mathrm{l}_{3}(0.1)\right\}$ & $\left\{l_{3}(1)\right\}$ & $\left\{l_{3}(1)\right\}$ & $\left\{l_{1}(0.5), l_{2}(0.5)\right\}$ \\
\hline $\mathrm{A}_{3}$ & $\left\{l_{3}(1)\right\}$ & $\left\{1_{1}(0.8), 1_{2}(0.2)\right\}$ & $\left\{l_{1}(0.8), l_{2}(0.2)\right\}$ & $\left\{1_{2}(0.4), 1_{3}(0.6)\right\}$ & $\left\{l_{1}(0.8), l_{2}(0.2)\right\}$ \\
\hline $\mathrm{A}_{4}$ & $\left\{1_{2}(0.8), 1_{3}(0.2)\right\}$ & $\left\{1_{1}(0.4), 1_{2}(0.6)\right\}$ & $\left\{l_{2}(0.9), l_{2}(0.1)\right\}$ & $\left\{1_{1}(0.2), 1_{2}(0.8)\right\}$ & $\left\{l_{2}(0.9), l_{3}(0.1)\right\}$ \\
\hline $\mathrm{A}_{5}$ & $\left\{1_{-1}(0.2), 1_{0}(0.8)\right\}$ & $\left\{1_{-2}(1)\right\}$ & $\left\{1_{-3}(0.2), 1_{-2}(0.8)\right\}$ & $\left\{1_{-2}(0.1), 1_{0}(0.9)\right\}$ & $\left\{1_{0}(0.8), 1_{1}(0.2)\right\}$ \\
\hline
\end{tabular}

\begin{tabular}{llllll}
\hline & $\mathrm{C}_{1}$ & $\mathrm{C}_{2}$ & $\mathrm{C}_{3}$ & $\mathrm{C}_{4}$ & $\mathrm{C}_{5}$ \\
\hline $\mathrm{A}_{1}$ & $\left\{\mathrm{l}_{2}(0.8), \mathrm{l}_{3}(0.2)\right\}$ & $\left\{\mathrm{l}_{3}(1)\right\}$ & $\left\{\mathrm{l}_{2}(0.1), 1_{3}(0.9)\right\}$ & $\left\{\mathrm{l}_{1}(0.8), \mathrm{l}_{2}(0.2)\right\}$ & $\left\{\mathrm{l}_{2},(0.1), 1_{3}(0.9)\right\}$ \\
$\mathrm{A}_{2}$ & $\left\{\mathrm{l}_{2}(0.5), \mathrm{l}_{3}(0.5)\right\}$ & $\left\{\mathrm{l}_{2}(0.5), \mathrm{l}_{3}(0.5)\right\}$ & $\left\{\mathrm{l}_{2}(0.8), 1_{3}(0.2)\right\}$ & $\left\{\mathrm{l}_{3}(1)\right\}$ & $\left\{\mathrm{l}_{2}(1)\right\}$ \\
$\mathrm{A}_{3}$ & $\left\{\mathrm{l}_{1}(0.7), \mathrm{l}_{2}(0.3)\right\}$ & $\left\{\mathrm{l}_{1}(0.6), \mathrm{l}_{2}(0.4)\right\}$ & $\left\{\mathrm{l}_{0}(0.3), \mathrm{l}_{1}(0.7)\right\}$ & $\left\{\mathrm{l}_{2}(0.5), \mathrm{l}_{3}(0.5)\right\}$ & $\left\{\mathrm{l}_{1}(0.5), \mathrm{l}_{2}(0.5)\right\}$ \\
$\mathrm{A}_{4}$ & $\left\{\mathrm{l}_{3}(1)\right\}$ & $\left\{\mathrm{l}_{2}(0.8), \mathrm{l}_{3}(0.2)\right\}$ & $\left\{\mathrm{l}_{1}(0.5), \mathrm{l}_{2}(0.5)\right\}$ & $\left\{\mathrm{l}_{0}(0.6), \mathrm{l}_{2}(0.4)\right\}$ & $\left\{\mathrm{l}_{2}(0.8), \mathrm{l}_{3}(0.2)\right\}$ \\
$\mathrm{A}_{5}$ & $\left\{\mathrm{l}_{0}(0.5), \mathrm{l}_{1}(0.5)\right\}$ & $\left\{\mathrm{l}_{1}(1)\right\}$ & $\left\{\mathrm{l}_{-2}(0.5), \mathrm{l}_{1}(0.5)\right\}$ & $\left\{\mathrm{l}_{1}(0.9), \mathrm{l}_{0}(0.1)\right\}$ & $\left\{\mathrm{l}_{0}(0.3), \mathrm{l}_{1}(0.7)\right\}$ \\
\hline
\end{tabular}

Table 3 The probabilistic linguistic decision matrix for the year 2015.

Table 4 The probabilistic linguistic decision matrix for the year 2019 .

\begin{tabular}{llllll}
\hline & $\mathrm{C}_{1}$ & $\mathrm{C}_{2}$ & $\mathrm{C}_{3}$ & $\mathrm{C}_{4}$ & $\mathrm{C}_{5}$ \\
\hline $\mathrm{A}_{1}$ & $\left\{\mathrm{l}_{2}(0.5), \mathrm{l}_{3}(0.5)\right\}$ & $\left\{\mathrm{l}_{3}(1)\right\}$ & $\left\{\mathrm{l}_{2}(0.2), \mathrm{l}_{3}(0.8)\right\}$ & $\left\{1_{1}(0.2), \mathrm{l}_{2}(0.8)\right\}$ & $\left\{\mathrm{l}_{2}(0.2), \mathrm{l}_{3}(0.8)\right\}$ \\
$\mathrm{A}_{2}$ & $\left\{\mathrm{l}_{2}(0.9), \mathrm{l}_{3}(0.1)\right\}$ & $\left\{\mathrm{l}_{2}(0.8), \mathrm{l}_{3}(0.2)\right\}$ & $\left\{\mathrm{l}_{2}(0.5), 1_{3}(0.5)\right\}$ & $\left\{\mathrm{l}_{3}(1)\right\}$ & $\left\{1_{1}(0.5), \mathrm{l}_{2}(0.5)\right\}$ \\
$\mathrm{A}_{3}$ & $\left\{\mathrm{l}_{0}(0.2), \mathrm{l}_{1}(0.8)\right\}$ & $\left\{\mathrm{l}_{1}(1)\right\}$ & $\left\{\mathrm{l}_{0}(0.2), 1_{1}(0.8)\right\}$ & $\left\{\mathrm{l}_{2}(0.5), \mathrm{l}_{3}(0.5)\right\}$ & $\left\{\mathrm{l}_{2}(0.8), \mathrm{l}_{3}(0.2)\right\}$ \\
$\mathrm{A}_{4}$ & $\left\{\mathrm{l}_{3}(1)\right\}$ & $\left\{1_{1}(0.7), \mathrm{l}_{2}(0.3)\right\}$ & $\left\{1_{1}(0.6), \mathrm{l}_{2}(0.4)\right\}$ & $\left\{1_{1}(0.7), \mathrm{l}_{2}(0.3)\right\}$ & $\left\{1_{1}(0.3), \mathrm{l}_{2}(0.7)\right\}$ \\
\hline
\end{tabular}




\begin{tabular}{llllll}
\hline $\mathrm{A}_{5}$ & $\left\{\mathrm{1}_{-2}(0.3), \mathrm{L}_{-1}(0.7)\right\}$ & $\left\{1_{-1}(0.2), \mathrm{l}_{0}(0.8)\right\}$ & $\left\{1_{-2}(0.1), \mathrm{l}_{0}(0.9)\right\}$ & $\left\{1_{0}(0.9), 1_{1}(0.1)\right\}$ & $\left\{1_{1}(1)\right\}$ \\
\hline
\end{tabular}

Table 5 The probabilistic linguistic decision matrix after being split for $\mathrm{C}_{1}$ (Li et al. 2020).

\begin{tabular}{|c|c|c|c|c|c|}
\hline & $A_{1}$ & $\mathrm{~A}_{2}$ & $\mathrm{~A}_{3}$ & $\mathrm{~A}_{4}$ & $\mathrm{~A}_{5}$ \\
\hline 2007 & $\begin{array}{l}\left\{1_{1}(0.3), 1_{2}(0.2),\right. \\
\left.1_{2}(0.3), 1_{2}(0.2)\right\}\end{array}$ & $\begin{array}{l}\left\{1_{1}(0.5), 1_{1}(0.3),\right. \\
\left.1_{2}(0.1), 1_{2}(0.1)\right\}\end{array}$ & $\begin{array}{l}\left\{1_{0}(0.2), 1_{0}(0.3),\right. \\
\left.1_{1}(0.2), 1_{1}(0.3)\right\}\end{array}$ & $\begin{array}{l}\left\{1_{2}(0.2), 1_{3}(0.6),\right. \\
\left.1_{3}(0.2)\right\}\end{array}$ & $\begin{array}{l}\left\{1_{-1}(0.2), 1_{-1}(0.1)\right. \\
\left.1_{-1}(0.1), 1_{0}(0.1), 1_{0}(0.5)\right\}\end{array}$ \\
\hline 2011 & $\begin{array}{l}\left\{1_{2}(0.3), 1_{2}(0.2),\right. \\
\left.1_{2}(0.3), 1_{3}(0.2)\right\}\end{array}$ & $\begin{array}{l}\left\{1_{2}(0.5), 1_{3}(0.3),\right. \\
\left.1_{3}(0.1), 1_{3}(0.1)\right\}\end{array}$ & $\begin{array}{l}\left\{1_{1}(0.2), 1_{1}(0.3),\right. \\
\left.1_{1}(0.2), 1_{2}(0.3)\right\}\end{array}$ & $\begin{array}{l}\left\{1_{3}(0.2), 1_{3}(0.6),\right. \\
\left.1_{3}(0.2)\right\}\end{array}$ & $\begin{array}{l}\left\{1_{0}(0.2), 1_{0}(0.1)\right. \\
\left.1_{0}(0.1), 1_{0}(0.1), 1_{1}(0.5)\right\}\end{array}$ \\
\hline 2015 & $\begin{array}{l}\left\{1_{2}(0.3), 1_{2}(0.2),\right. \\
\left.1_{2}(0.3), 1_{2}(0.2)\right\}\end{array}$ & $\begin{array}{l}\left\{1_{1}(0.5), 1_{2}(0.3),\right. \\
\left.1_{2}(0.1), l_{2}(0.1)\right\}\end{array}$ & $\begin{array}{l}\left\{1_{3}(0.2), 1_{3}(0.3),\right. \\
\left.1_{3}(0.2), 1_{3}(0.3)\right\}\end{array}$ & $\begin{array}{l}\left\{1_{2}(0.2), 1_{2}(0.6),\right. \\
\left.1_{3}(0.2)\right\}\end{array}$ & $\begin{array}{l}\left\{1_{-1}(0.2), 1_{0}(0.1)\right. \\
\left.1_{0}(0.1), 1_{0}(0.1), 1_{0}(0.5)\right\}\end{array}$ \\
\hline 2019 & $\begin{array}{l}\left\{\mathrm{l}_{2}(0.3), \mathrm{l}_{2}(0.2),\right. \\
\left.\mathrm{l}_{3}(0.3), \mathrm{l}_{3}(0.2)\right\}\end{array}$ & $\begin{array}{l}\left\{l_{2}(0.5), 1_{2}(0.3),\right. \\
\left.l_{2}(0.1), 1_{3}(0.1)\right\}\end{array}$ & $\begin{array}{l}\left\{1_{0}(0.2), 1_{1}(0.3),\right. \\
\left.1_{1}(0.2), 1_{1}(0.3)\right\}\end{array}$ & $\begin{array}{l}\left\{1_{3}(0.2), 1_{3}(0.6),\right. \\
\left.1_{3}(0.2)\right\}\end{array}$ & $\begin{array}{l}\left\{1_{-2}(0.2), 1_{-2}(0.1),\right. \\
\left.1_{-1}(0.1), 1_{-1}(0.1), 1_{-1}(0.5)\right\}\end{array}$ \\
\hline
\end{tabular}

Table 6 The hesitant probabilistic fuzzy decision matrix calculated by formula (8) for $\mathrm{C}_{1}$.

\begin{tabular}{llllll}
\hline & $\mathrm{A}_{1}$ & $\mathrm{~A}_{2}$ & $\mathrm{~A}_{3}$ & $\mathrm{~A}_{4}$ & $\mathrm{~A}_{5}$ \\
\hline 2007 & $\{2 / 3(0.3), 2 / 3(0.2)$, & $\{2 / 3(0.5), 2 / 3(0.3)$, & $\{1 / 2(0.2), 1 / 2(0.3)$, & $\{5 / 6(0.2), 1(0.6)$, & $\{1 / 3(0.2), 1 / 3(0.1)$, \\
& $5 / 6(0.3), 5 / 6(0.2)\}$ & $5 / 6(0.1), 5 / 6(0.1)\}$ & $2 / 3(0.2), 2 / 3(0.3)\}$ & $1(0.2)\}$ & $1 / 3(0.1), 1 / 2(0.1), 1 / 2(0.5)\}$ \\
2011 & $\{5 / 6(0.3), 5 / 6(0.2)$, & $\{5 / 6(0.5), 1(0.3)$, & $\{2 / 3(0.2), 2 / 3(0.3)$, & $\{1(0.2), 1(0.6)$, & $\{1 / 2(0.2), 1 / 2(0.1)$, \\
& $5 / 6(0.3), 1(0.2)\}$ & $1(0.1), 1(0.1)\}$ & $2 / 3(0.2), 5 / 6(0.3)\}$ & $1(0.2)\}$ & $1 / 2(0.1), 1 / 2(0.1), 1 / 2(0.5)\}$ \\
2015 & $\{5 / 6(0.3), 5 / 6(0.2)$, & $\{2 / 3(0.5), 5 / 6(0.3)$, & $\{1(0.2), 1(0.3)$, & $\{5 / 6(0.2), 5 / 6(0.6)$, & $\{1 / 3(0.2), 1 / 2(0.1)$, \\
& $5 / 6(0.3), 5 / 6(0.2)\}$ & $5 / 6(0.1), 5 / 6(0.1)\}$ & $1(0.2), 1(0.3)\}$ & $1(0.2)\}$ & $1 / 2(0.1), 1 / 2(0.1), 1 / 2(0.5)\}$ \\
2019 & $\{5 / 6(0.3), 5 / 6(0.2)$, & $\{5 / 6(0.5), 5 / 6(0.3)$, & $\{1 / 2(0.2), 2 / 3(0.3)$, & $\{1(0.2), 1(0.6)$, & $\{1 / 6(0.2), 1 / 6(0.1)$, \\
& $1(0.3), 1(0.2)\}$ & $5 / 6(0.1), 1(0.1)\}$ & $2 / 3(0.2), 2 / 3(0.3)\}$ & $1(0.2)\}$ & $1 / 3(0.1), 1 / 3(0.1), 1 / 3(0.5)\}$ \\
\hline
\end{tabular}

Table 7 The hesitant probabilistic fuzzy decision matrix.

\begin{tabular}{llllll}
\hline & $\mathrm{C}_{1}$ & $\mathrm{C}_{2}$ & $\mathrm{C}_{3}$ & $\mathrm{C}_{4}$ & $\mathrm{C}_{5}$ \\
\hline $\mathrm{A}_{1}$ & $\{0.961(0.3), 0.972(0.2)$, & $\{1(1)\}$ & $\{1(1)\}$ & $\{0.889(0.2), 0.921(0.4)$, & $\{0.972(0.1), 1(0.9)\}$ \\
& $1(0.5)\}$ & & $0.944(0.2), 0.961(0.2)\}$ & \\
$\mathrm{A}_{2}\{0.944(0.5), 1(0.5)\}$ & $\{0.961(0.2), 0.972(0.3)$, & $\{1(1)\}$ & $\{1(1)\}$ & $\{0.944(0.5), 0.972(0.4)$, \\
& & $1(0.5)\}$ & & & $1(0.1)\}$ \\
$\mathrm{A}_{3}\{1(1)\}$ & $\{0.889(0.6), 0.944(0.2)$, & $\{0.833(0.2), 0.864(0.1)$, & $\{0.944(0.2), 0.972(0.2)$, & $\{0.921(0.4), 0.944(0.1)$, \\
& & $0.961(0.2)\}$ & $0.889(0.5), 0.944(0.2)\}$ & $1(0.6)\}$ & $0.961(0.3), 1(0.2)\}$ \\
$\mathrm{A}_{4}\{1(1)\}$ & $\{0.944(0.4), 0.961(0.3)$, & $\{0.944(0.5), 0.961(0.1)$, & $\{0.864(0.2), 0.904(0.4)$, & $\{0.961(0.3), 0.972(0.4)$, \\
& & $0.972(0.1), 1(0.2)\}$ & $0.972(0.1), 1(0.3)\}$ & $0.944(0.1), 0.961(0.1), 0.972(0.2)\}$ & $1(0.3)\}$ \\
$\mathrm{A}_{5}\{0.570(0.2), 0.627(0.1)$, & $\{0.444(0.2), 0.627(0.8)\}$ & $\{0.741(0.1), 0.876(0.1)$, & $\{0.519(0.1), 0.938(0.8)$, & $\{0.833(0.3), 0.864(0.5)$, \\
& $0.667(0.1), 0.711(0.6)\}$ & & $0.897(0.3), 0.917(0.5)\}$ & $0.969(0.1)\}$ & $0.944(0.2)\}$ \\
\hline
\end{tabular}

Table 8 The probabilistic linguistic decision matrix.

\begin{tabular}{llllll}
\hline & $\mathrm{C}_{1}$ & $\mathrm{C}_{2}$ & $\mathrm{C}_{3}$ & $\mathrm{C}_{4}$ & $\mathrm{C}_{5}$ \\
\hline $\mathrm{A}_{1}$ & $\left\{1_{2.766}(0.3), 1_{2.832}(0.2)\right.$, & $\left\{1_{3}(1)\right\}$ & $\left\{1_{3}(1)\right\}$ & $\left\{1_{2.334}(0.2), 1_{2.526}(0.4)\right.$, & $\left\{1_{2.832}(0.1), 1_{3}(0.9)\right\}$ \\
& $\left.1_{3}(0.5)\right\}$ & & $\left.1_{2.664}(0.2), 1_{2.766}(0.2)\right\}$ & \\
$\mathrm{A}_{2}$ & $\left\{1_{2.664}(0.5), 1_{3}(0.5)\right\}$ & $\left\{1_{2.766}(0.2), 1_{2.832}(0.3)\right.$, & $\left\{1_{3}(1)\right\}$ & $\left\{1_{3}(1)\right\}$ & $\left\{1_{2.664}(0.5), 1_{2.832}(0.4)\right.$, \\
& & $\left.1_{3}(0.5)\right\}$ & & & $\left.1_{3}(0.1)\right\}$ \\
$\mathrm{A}_{3}$ & $\left\{1_{3}(1)\right\}$ & $\left\{1_{2.334}(0.6), 1_{2.664}(0.2)\right.$, & $\left\{1_{1.998}(0.2), 1_{2.184}(0.1)\right.$, & $\left\{1_{2.664}(0.2), 1_{2.832}(0.2)\right.$, & $\left\{1_{2.526}(0.4), 1_{2.664}(0.1)\right.$, \\
\hline
\end{tabular}




\begin{tabular}{llllll}
\hline & & $\left.1_{2.766}(0.2)\right\}$ & $\left.1_{2.334}(0.5), 1_{2.664}(0.2)\right\}$ & $\left.1_{3}(0.6)\right\}$ & $\left.1_{2.766}(0.3), l_{3}(0.2)\right\}$ \\
$\mathrm{A}_{4}$ & $\left\{1_{3}(1)\right\}$ & $\left\{1_{2.664}(0.4), 1_{2.766}(0.3)\right.$, & $\left\{1_{2.664}(0.5), 1_{2.766}(0.1)\right.$, & $\left\{1_{2.184}(0.2), 1_{2.424}(0.4)\right.$, & $\left\{1_{2.766}(0.3), 1_{2.832}(0.4)\right.$, \\
& & $\left.1_{2.832}(0.1), 1_{3}(0.2)\right\}$ & $\left.1_{2.832}(0.1), 1_{3}(0.3)\right\}$ & $\left.1_{2.664}(0.1), 1_{2.766}(0.1), 1_{2.832}(0.2)\right\}$ & $\left.1_{3}(0.3)\right\}$ \\
$\mathrm{A}_{5} \quad\left\{1_{0.42}(0.2), 1_{0.762}(0.1)\right.$, & $\left\{1_{-0.336}(0.2), 1_{0.762}(0.8)\right\}$ & $\left\{1_{1.446}(0.1), 1_{2.256}(0.1)\right.$, & $\left\{1_{0.114}(0.1), 1_{2.628}(0.8)\right.$, & $\left\{1_{1.998}(0.3), 1_{2.184}(0.5)\right.$, \\
& & & $\left.1_{2.382}(0.3), 1_{2.502}(0.5)\right\}$ & $\left.1_{2.814}(0.1)\right\}$ & $\left.1_{2.664}(0.2)\right\}$ \\
\hline
\end{tabular}

Table 9 Parameters values.

\begin{tabular}{llllll}
\hline Parameter & $\mathrm{C}_{1}$ & $\mathrm{C}_{2}$ & $\mathrm{C}_{3}$ & $\mathrm{C}_{4}$ & $\mathrm{C}_{5}$ \\
\hline $\mathrm{q}$ & 1.1 & 1 & 0.9 & 1.2 & 0.9 \\
$\mathrm{p}$ & 1.2 & 1.5 & 1.1 & 1.4 & 1.3 \\
$\mathrm{v}$ & 1.3 & 2 & 1.3 & 1.6 & 1.5 \\
\hline
\end{tabular}

Table 10 Experimental results of the four methods

\begin{tabular}{ll}
\hline Methods & Ranking \\
\hline PL-MAUT-PAMSSEM II & $\mathrm{A}_{1}>\mathrm{A}_{2}>\mathrm{A}_{4}>\mathrm{A}_{3}>\mathrm{A}_{5}$ \\
MAUT & $\mathrm{A}_{2}>\mathrm{A}_{1}>\mathrm{A}_{4}>\mathrm{A}_{3}>\mathrm{A}_{5}$ \\
PAMSSEM II & $\mathrm{A}_{4}>\mathrm{A}_{5}>\mathrm{A}_{1}>\mathrm{A}_{2}>\mathrm{A}_{3}$ \\
PROMETHEE II (Brans et al. 1986; Figueira et al. 2005) & $\mathrm{A}_{1}>\mathrm{A}_{3}=\mathrm{A}_{4}>\mathrm{A}_{2}>\mathrm{A}_{5}$ \\
TOPSIS (Zhao et al. 2016) & $\mathrm{A}_{2}>\mathrm{A}_{1}>\mathrm{A}_{4}>\mathrm{A}_{3}>\mathrm{A}_{5}$ \\
ARAS (Zhu et al. 2012) & $\mathrm{A}_{2}>\mathrm{A}_{1}>\mathrm{A}_{4}>\mathrm{A}_{3}>\mathrm{A}_{5}$ \\
\hline
\end{tabular}

The same parameters values and weight are applied to the MAUT, PAMSSEM II, PROMETHEE, TOPSIS, and ARAS methods to get the different ranking. We show all the ranking results in Table 10. It can be seen from the table that when different decision-making methods are applied to MAGDM problems, they do not always get the same ranking results. Judging from the actual development of Sichuan, Chongqing, Guizhou, Yunnan, and Tibet in recent years, when all evaluation indicators are objective, the decision-making results given by the PL-MAUT-PAMSSEM II method are more realistic. Therefore, the comparison of various methods can more highlight the objectivity and rationality of the PL-MAUT-PAMSSEM II method proposed in this paper in solving practical problems.

\section{Conclusions}

PLTS is composed of several possible linguistic terms and their relative distribution, and it is an important means of expressing DM's wishes or evaluation in a linguistic environment. Due to its complex structure, PLTS is suitable 
for expressing qualitative evaluation information in the actual decision-making process, but its application in the calculation is limited. Therefore, we combine PLTS, HPFS, and scoring functions. In this article, the PL-MAUT-PAMSSEM II method is based on different criteria to compare the marginal utility scores of the alternatives in pairs, combining MAUT and PAMSSEM II and applying them to the selection of inbound tourist attractions. It is the advantage of the MAUT method to express the preference of the DMs by introducing a utility function. The utility function is composed of various attributes, which can evaluate the total utility of alternatives. For each attribute, the DMs will give an evaluation value. After the evaluation value is standardized to the interval $[0,1]$, the score value calculated by the marginal utility function is called the marginal utility score. After the calculation of the marginal utility score, we added the PAMSSEM II method as the second stage of data processing. The purpose of this method is to build a priority relationship and use the marginal utility score to establish a local preference model. We calculate the consistency index and the inconsistency index by comparing the alternatives in pairs, and then perform a weighted summary on them, and obtain the final ranking of the scheme by calculating the net dominant flow. The PAMSSEM method has significant advantages in dealing with mixed data (cardinal and ordinal evaluation values) and missing data (irrelevant evaluation values or evaluation values that are related but difficult to obtain). In addition, this method can not only distinguish between different attributes Thresholds (preference threshold and irrelevant threshold) represent the degree of preference of the DM, and the rejection threshold can also be used to represent the compensation limit that the DM is willing to accept. Therefore, the results calculated by the combined application of MAUT and PAMSSEM are more convincing.

We will broaden the application areas of the MAUT-PAMSSEM II method, such as applying the method to project renovation selection, contractor selection, investment evaluation, and risk evaluation in the future. At the same time, we will further study other special cases of MAUT-PAMSSEM II in a probabilistic language environment. Of course, we will combine MAUT-PAMSSEM II with some information aggregation operators and 
apply them to other fuzzy environments, such as dual hesitation fuzzy sets (Farhadinia and Xu 2016; Meng et al. 2015), language interval hesitation fuzzy sets (Lin et al. 2021), and weighted ordered hesitation fuzzy set (Zavadskas et al. 2010).

Funding This study was funded by the National Social Science Foundation of China (Grant No. 16CGL026).

\section{Compliance with ethical standards}

Conflict of interest The authors declare that they have no conflict of interest.

Ethical approval This article does not contain any studies with human participants or animals performed by any of the authors.

\section{References}

Alinezhad A and Khalili J (2019) New methods and applications in multiple attribute decision making (madm). International Series in Operations Research \& Management Science 277:157-165

Bai CZ, Zhang R, Qian LX and Wu YN (2017) Comparisons of probabilistic linguistic term sets for multi-criteria decision making. Knowledge-Based Systems 119:284-291

Bélanger M, Guitouni A and Hunter C (2000a) Advances in intelligent systems: Theory and applications. IOS press 59:302-310

Bélanger M, Guitouni A and Hunter C (2000b) Coa advisory system based on the multiple criteria decision analysis. Defence Reserch Establishment Valcartier,

Bélanger M and Martel JM (2012) Explanations for a decision support system based on mcda. Computing and Informatics 25:195-221

Brans JP, Vincke Ph and Mareschal B (1986) How to select and how to rank projects: The promethee method. European Journal of Operational Research 24:228-238

Diakoulaki D, Mavrotas G and Papayannakis L (1995) Determining objective weights in multiple criteria problems: The critic method. Computers \& Operations Research 22:763-770

Emovon I, Norman RA and Murphy AJ (2016) An integration of multi-criteria decision making techniques with a delay time model for determination of inspection intervals for marine machinery systems. Applied Ocean Research 59:65-82

Farhadinia B and Xu ZS (2016) Distance and aggregation-based methodologies for hesitant fuzzy decision making. Cognitive Computation 9:81-94

Feng XQ, Liu Q and Wei CP (2019) Probabilistic linguistic qualiflex approach with possibility degree comparison. Journal of Intelligent \& Fuzzy Systems 36:719-730

Figueira J, Greco S and Ehrgott M (2005) Multiple criteria decision analysis. Springer http://doi.org/10.1007/b100605 
Gao J, Xu ZS, Ren PJ and Liao HC (2018) An emergency decision making method based on the multiplicative consistency of probabilistic linguistic preference relations. International Journal of Machine Learning and Cybernetics 10:1613-1629

Gou XJ and Xu ZS (2016) Novel basic operational laws for linguistic terms, hesitant fuzzy linguistic term sets and probabilistic linguistic term sets. Information Sciences 372:407-427

Gou XJ, Xu ZS and Liao HC (2016) Multiple criteria decision making based on bonferroni means with hesitant fuzzy linguistic information. Soft Computing 21:6515-6529

Guitouni A, Martel JM, Belanger M and Hunter C (1999) Managing a decision making situation in the context of the canadian airspace protection. Endocrinology 44:409-414

Hafezalkotob A and Hafezalkotob A (2015) Comprehensive multimoora method with target-based attributes and integrated significant coefficients for materials selection in biomedical applications. Materials \& Design 87:949-959

He YH, Guo HW, Jin MZ and Ren PY (2016) A linguistic entropy weight method and its application in linguistic multi-attribute group decision making. Nonlinear Dynamics 84:399-404

Herrera F, Herrera-Viedma E and Martinez L (2000) A fusion approach for managing multi-granularity linguistic term sets in decision making. Fuzzy Sets \& Systems 114:43-58

Herrera F, Herrera-Viedma E and Martinez L (2008) A fuzzy linguistic methodology to deal with unbalanced linguistic term sets. IEEE Transactions on Fuzzy Systems 16:354-370

Ji ZJ and Yu HS (2017) A new perspective to graphical characterization of multiagent controllability. IEEE Trans Cybern 47:1471-1483

Li J, Chen QX, Niu LL and Wang ZX (2020) An oreste approach for multi-criteria decision-making with probabilistic hesitant fuzzy information. International Journal of Machine Learning and Cybernetics 11:1591-1609

Liao HC, Mi XM and Xu ZS (2019) A survey of decision-making methods with probabilistic linguistic information: Bibliometrics, preliminaries, methodologies, applications and future directions. Fuzzy Optimization and Decision Making 19:81-134

Liao HC, Xu ZS, Zeng XJ and Merigó JM (2015) Qualitative decision making with correlation coefficients of hesitant fuzzy linguistic term sets. Knowledge-Based Systems 76:127-138

Lin MW, Chen ZY, Liao HC and Xu ZS (2019) Electre ii method to deal with probabilistic linguistic term sets and its application to edge computing. Nonlinear Dynamics 96:2125-2143

Lin MW, Chen ZY, Xu ZS, Gou XJ and Herrera F (2021) Score function based on concentration degree for probabilistic linguistic term sets: An application to topsis and vikor. Information Sciences 551:270-290

Lin MW and Xu ZS (2018) Probabilistic linguistic distance measures and their applications in multi-criteria group decision making. Fuzziness and Soft Computing 357:411-440

Lootsma FA (2010) Scale sensitivity in the multiplicative ahp and smart. Journal of Multi-Criteria Decision Analysis 2:87-110

Martel JM (1998) Multicriterion analysis under uncertainty: The approach of outranking synthesis. Papers,

Meng FY, Wang C and Chen XH (2015) Linguistic interval hesitant fuzzy sets and their application in decision making. Cognitive Computation 8:52-68

Mi XM, Liao HC, Wu XL and Xu ZS (2020) Probabilistic linguistic information fusion: A survey on aggregation operators in terms of principles, definitions, classifications, applications, and challenges. International Journal of Intelligent Systems 35:529-556

Nemery P and Ishizaka A (2013) Multi-criteria decision analysis: Methods and software. Computer Science, http://doi.org/10.1002/9781118644898 
Pang Q, Wang H and Xu ZS (2016) Probabilistic linguistic term sets in multi-attribute group decision making. Information Sciences 369:128-143

Qu JJ, Ji ZJ, Lin C and Yu HS (2018) Fast consensus seeking on networks with antagonistic interactions. Complexity 2018:1-15

Rodríguez A, Ortega F and Concepción R (2017) An intuitionistic method for the selection of a risk management approach to information technology projects. Information Sciences 375:202-218

Rodriguez RM, Martinez L and Herrera F (2012) Hesitant fuzzy linguistic term sets for decision making. IEEE Transactions on Fuzzy Systems 20:109-119

Saleem A and NoorUl A (2014) Analysis of s-box image encryption based on generalized fuzzy soft expert set. Nonlinear Dynamics 79:1679-1692

Song YM and Jun H (2019) Large-scale group decision making with multiple stakeholders based on probabilistic linguistic preference relation. Applied Soft Computing 80:712-722

Sun KK, Mou SS, Qiu JB, Wang T and Gao HJ (2019) Adaptive fuzzy control for nontriangular structural stochastic switched nonlinear systems with full state constraints. IEEE Transactions on Fuzzy Systems 27:1587-1601

Tian ZP, Nie RX and Wang JQ (2019) Probabilistic linguistic multi-criteria decision-making based on evidential reasoning and combined ranking methods considering decision-makers' psychological preferences. Journal of the Operational Research Society 71:700-717

Torra V (2010) Hesitant fuzzy sets. International Journal of Intelligent Systems 25:529-539

Torra V and Narukawa Y (2009) On hesitant fuzzy sets and decision. IEEE International Conference on Fuzzy Systems, http://doi.org/10.1109/FUZZY.2009.5276884

Wallenius J, Fishburn PC, Zionts S, Dyer JS, Steuer RE and Deb K (2008) Multiple criteria decision making, multiattribute utility theory: Recent accomplishments and what lies ahead. INFORMS 54:1336-1349

Wang H, Xu ZS and Zeng XJ (2018a) Modeling complex linguistic expressions in qualitative decision making: An overview. Knowledge-Based Systems 144:174-187

Wang JQ, Peng L, Zhang HY and Chen XH (2014) Method of multi-criteria group decision-making based on cloud aggregation operators with linguistic information. Information Sciences 274:177-191

Wang Q, Wu C and Sun Y (2015) Evaluating corporate social responsibility of airlines using entropy weight and grey relation analysis. Journal of Air Transport Management 42:55-62

Wang XK, Wang JQ and Zhang HY (2018b) Distance-based multicriteria group decision-making approach with probabilistic linguistic term sets. Expert Systems, https://oi.org/10.1111/exsy.12352

Wu LP, Hu JM and Zhang J (2020) The spatial econometric modeling and influencing factor effects of inbound tourism development in china's provincial regions. Tourism Tribune 35:14-27

Wu XL, Liao HC, Xu ZS, Hafezalkotob A and Herrera F (2018) Probabilistic linguistic multimoora: A multicriteria decision making method based on the probabilistic linguistic expectation function and the improved borda rule. IEEE Transactions on Fuzzy Systems 26:3688-3702

Xi JX, Wang C, Liu H and Wang L (2019) Completely distributed guaranteed-performance consensualization for high-order multiagent systems with switching topologies. IEEE Trans. Syst. Man Cybern. -Syst. 49:1338-1348

Xi JX, Wang C, Liu H and Wang Z (2018) Dynamic output feedback guaranteed-cost synchronization for multiagent networks with given cost budgets. IEEE Access 6:28923-28935

Xia MM and Xu ZS (2011) Hesitant fuzzy information aggregation in decision making. International Journal of Approximate Reasoning 52:395-407

Xian SD, Chai JH and Yin YB (2019) A visual comparison method and similarity measure for probabilistic 
linguistic term sets and their applications in multi-criteria decision making. International Journal of Fuzzy Systems 21:1154-1169

Xu WZ, Fang J and Liu XK (2021) Research on evaluation of urban road resources and environmental benefits based on entropy method. THE WORLD OF BUILDING MATERIALS, http://doi.org/10.3963/j.issn.1674-6066.2021.02.028

Xu ZS (2004) Uncertain linguistic aggregation operators based approach to multiple attribute group decision making under uncertain linguistic environment. Information Sciences 168:171-184

Xu ZS (2008) An interactive approach to multiple attribute group decision making with multigranular uncertain linguistic information. Group Decision and Negotiation 18:119-145

Xu ZS and Zhang S (2021) An overview of probabilistic hesitant fuzzy decision-making theory and methods. Control and Decision, https://doi.org/10.13195/j.kzyjc.2020.0465

$\mathrm{Xu}$ ZS and Zhou W (2016) Consensus building with a group of decision makers under the hesitant probabilistic fuzzy environment. Fuzzy Optimization and Decision Making 16:481-503

Yang GH and Cui B (2011) The application of entropy weight method to evaluation of the sustainable utilization of water resources. Mathematics in Practice and Theory 41:8-12

Yazir YO, Akbulut Y, Farahbod R, Guitouni A and Coady Y (2012) Autonomous resource consolidation management in clouds using impromptu extensions. IEEE International Conference on Cloud Computing 5:614-621

Yu WW, Zhang H and Li BQ (2019a) Comparison and operators based on uncertain probabilistic linguistic term set. Journal of Intelligent \& Fuzzy Systems 36:6359-6379

Yu WW, Zhang H and Li BQ (2019b) Operators and comparisons of probabilistic linguistic term sets. International Journal of Intelligent Systems 34:1476-1504

Yue N, Xie JL and Chen SL (2020) Some new basic operations of probabilistic linguistic term sets and their application in multi-criteria decision making. Soft Computing 24:12131-12148

Zadeh LA (1975) The concept of a linguistic variable and its application to approximate reasoning. Information Sciences 8:199-249

Zavadskas EK, Turskis Z and Vilutiene T (2010) Multiple criteria analysis of foundation instalment alternatives by applying additive ratio assessment (aras) method. Archives of Civil and Mechanical Engineering 10:123-141

Zhai YL, Xu ZS and Liao HC (2016) Probabilistic linguistic vector-term set and its application in group decision making with multi-granular linguistic information. Applied Soft Computing 49:801-816

Zhang JL and Qi XW (2013) Research on multiple attribute decision making under hesitant fuzzy linguistic environment with application to production strategy decision making. Advanced Materials Research 753-755:2829-2836

Zhang S, Xu ZS and He Y (2017) Operations and integrations of probabilistic hesitant fuzzy information in decision making. Information Fusion, http://doi.org/10.1016/j.inffus.2017.02.001

Zhang X, Liao H, Xu B and Xiong M (2020) A probabilistic linguistic-based deviation method for multi-expert qualitative decision making with aspirations. Applied Soft Computing, https://doi.org/10.1016/j.asoc.2020.106362

Zhang YX, Xu ZS and Liao HC (2018) An ordinal consistency-based group decision making process with probabilistic linguistic preference relation. Information Sciences 467:179-198

Zhang YX, Xu ZS, Wang H and Liao HC (2016) Consistency-based risk assessment with probabilistic linguistic preference relation. Applied Soft Computing 49:817-833

Zhao N, Xu ZS and Liu FJ (2016) Group decision making with dual hesitant fuzzy preference relations. Cognitive 
Computation 8:1119-1143

Zhu B, Xu ZS and Xia MM (2012) Dual hesitant fuzzy sets. Journal of Applied Mathematics 2012:1-13

Zou ZH, Yun Y and Sun JN (2006) Entropy method for determination of weight of evaluating indicators in fuzzy synthetic evaluation for water quality assessment. Journal of Environmental Sciences 18:1020-1023 